Elsevier Editorial System(tm) for Revista Científica de la Sociedad Española de Enfermería Neurológica Manuscript Draft

Manuscript Number:

Title: Intervención psicoeducativa para personas con Enfermedad de Parkinson y familiares cuidadores: resultados preliminares de la medición basal. Psychoeducational intervention for people with Parkinson's Disease and family carers: preliminary results at baseline time.

Article Type: Original

Keywords: Intervención multidisciplinar; habilidades de afrontamiento; adaptación psicosocial; cuidadores informales; enfermedad de Parkinson.

Multidisciplinary intervention; coping skills; psychosocial adjustment; informal caregivers; Parkinson's disease.

Corresponding Author: Dr. M Victoria Navarta-Sanchez, PhD

Corresponding Author's Institution:

First Author: M Victoria Navarta-Sanchez, PhD

Order of Authors: M Victoria Navarta-Sanchez, PhD; Mario Riverol; M Eugenia Ursúa; Leire Ambrosio; Juana M Senosiain; Mari Carmen Portillo

Abstract: INTRODUCCIÓN: La adaptación psicosocial influye en la calidad de vida del paciente con enfermedad de Parkinson (EP) y su familiar cuidador. Sin embargo, desde la práctica clínica generalmente no se aborda.

OBJETIVO: Evaluar habilidades de afrontamiento, adaptación psicosocial y calidad de vida, en tiempo basal, en pacientes con EP y familiares cuidadores de un cuasiexperimento.

MÉTODO: Estudio cuasiexperimental realizado en centros de Atención Primaria, con una muestra de 80 pacientes con EP y 80 familiares, distribuidos en un grupo control (GC) y un grupo experimental (GE). En la recogida de datos se utiliza la Escala de Adaptación psicosocial PAIS-SR, la Escala de Afrontamiento Brief Cope y las Escalas de Calidad de vida PDQ-39 y SQLC. En el análisis de datos sociodemográficos y T-student se utiliza SPSS 23.0 .

RESULTADOS: En el GC la edad media en pacientes fue 73,2 años, y en familiares 61,1 años. En el GE la edad media en pacientes fue 74,8 años, y en familiares 65,1 años. El 54\% y 40\% de los pacientes de ambos grupos presentaba Estadio I en Hoehn \& Yahr. Los pacientes y familiares del GC y GE presentaron una afectación leve de su calidad de vida y algunas dificultades en la adaptación psicosocial. Los pacientes y familiares del GC y GE utilizaban habilidades de afrontamiento con baja frecuencia. No se identificaron diferencias estadísticamente significativas entre GC y $\mathrm{GE}$.

CONCLUSIONES: Desde el diagnóstico se observan dificultades para la adaptación a la EP en pacientes y familiares, que podrían abordarse con intervenciones psicoeducativas multidisciplinares. 
INTRODUCTION: The psychosocial adjustment influences on quality of life in patient with Parkinson's disease (PD) and his/her family carer. However, this is usually not addressed in clinical practice. OBJECTIVE: To evaluate coping skills, psychosocial adjustment and quality of life in patients with PD and family carers from a quasiexperiment at baseline time.

METHODS: Quasi-experimental study carried out in Primary Care centres, with a sample of 80 patients with PD and 80 family carers, divided in a control group (CG) and an experimental group (EG). In the data collection was used the Psychosocial Adjustment Scale PAIS-SR, the Coping Scale Brief COPE and the Quality of Life Scales PDQ-39 and SQLC. The analysis of sociodemographic data and students t tests is performed with SPSS 23.0 .

RESULTS: In the CG the average age in patients was 73,2 years old, and in relatives 61,1 years old. In the $\mathrm{EG}$ the average age in patients was 74,8 years old, and in relatives 65, 1 years old. In both groups, 54\% and 40\% of patients had Hoehn \& Yahr stage I. Patients and relatives from CG and EG noticed mild difficulties in the quality of life and psychosocial adjustment to illness. Patients and relatives from CG and EG used the evaluated coping skills with low frequency. No statistically significant differences were found between CG and EG.

CONCLUSION: Since the diagnosis some difficulties are observed for the adjustment to PD in patients and relatives which could be addressed with multidisciplinary psychoeducational interventions. 


\section{PRIMERA PÁGINA}

\section{a) Título del artículo en castellano e inglés:}

Intervención psicoeducativa para personas con Enfermedad de Parkinson y familiares cuidadores: resultados preliminares de la medición basal.

Psychoeducational intervention for people with Parkinson's Disease and family carers: preliminary results at baseline time.

b) Nombre de pila y los dos apellidos de cada uno de los autores:

(1)M Victoria Navarta-Sánchez, (2)Mario Riverol, (3)M Eugenia Ursúa, (4)Leire Ambrosio, (5)Juana M Senosiain, (6)Mari Carmen Portillo

c) Filiación institucional (nombre completo del departamento, centro de trabajo y dirección completa del mismo)

(1) (4) (5) Departamento de Enfermería de la Persona Adulta. Facultad de Enfermería. Universidad de Navarra. C/ Irunlarrea, s/n, Edif. De los Castaños, 31008, Pamplona (Navarra), España.

(2) Departamento de Neurología, Clínica Universidad de Navarra. Avenida Pio XII, 36, 31008, Pamplona (Navarra), España.

(3) Centro de Salud de San Juan, Servicio Navarro de Salud-Osasunbidea. Plaza Obispo Irurita s/n Planta Baja, 31011, Pamplona (Navarra), España.

(6) Universidad de Southampton, Facultad de Ciencias de la Salud. Highfield Campus. Building 67 University Road Southampton, Hampshire SO17 1BJ, Reino Unido 
d) Nombre, filiación profesional, dirección de correo electrónico, y teléfono del autor responsable para la correspondencia

M Victoria Navarta-Sánchez. PhD. MSc. RN. Facultad de Enfermería. Universidad de Navarra.mnavarta@unav.es. Telf: +34948 425600

e) Financiación total o parcial del estudio si existiese

Este trabajo ha recibido financiación del Plan de Investigación de la Universidad de Navarra (PIUNA 2014-23).

\section{f) Agradecimientos}

Nuestro agradecimiento a los pacientes con Enfermedad de Parkinson y a las familias que han participado en el estudio por su tiempo y contribución en este proyecto, al equipo de la Línea de investigación de Procesos Crónicos y Cuidados al Final de la Vida de la Facultad de Enfermería de la Universidad de Navarra, y especialmente a los investigadores del Programa ReNACE.

g) Recuentos de palabras del

- resumen (en castellano y en inglés): 250 palabras en castellano / 244 inglés

- del cuerpo del manuscrito (Introducción, Método, Resultados, y Discusión): 2992 palabras 


\section{RESUMEN}

TíTULO: Intervención psicoeducativa para personas con enfermedad de Parkinson y familiares cuidadores: resultados preliminares de la medición basal.

INTRODUCCIÓN: La adaptación psicosocial influye en la calidad de vida del paciente con enfermedad de Parkinson (EP) y su familiar cuidador. Sin embargo, desde la práctica clínica generalmente no se aborda.

OBJETIVO: Evaluar habilidades de afrontamiento, adaptación psicosocial y calidad de vida, en tiempo basal, en pacientes con EP y familiares cuidadores de un cuasiexperimento.

MÉTODO: Estudio cuasiexperimental realizado en centros de Atención Primaria, con una muestra de 80 pacientes con EP y 80 familiares, distribuidos en un grupo control (GC) y un grupo experimental (GE). En la recogida de datos se utiliza la Escala de Adaptación psicosocial PAIS-SR, la Escala de Afrontamiento Brief Cope y las Escalas de Calidad de vida PDQ-39 y SQLC. En el análisis de datos sociodemográficos y Tstudent se utiliza SPSS 23.0.

RESULTADOS: En el GC la edad media en pacientes fue 73,2 años, y en familiares 61,1 años. En el GE la edad media en pacientes fue 74,8 años, y en familiares 65,1 años. El $54 \%$ y $40 \%$ de los pacientes de ambos grupos presentaba Estadio I en Hoehn \& Yahr. Los pacientes y familiares del GC y GE presentaron una afectación leve de su calidad de vida y algunas dificultades en la adaptación psicosocial. Los pacientes y familiares del GC y GE utilizaban habilidades de afrontamiento con baja frecuencia. No se identificaron diferencias estadísticamente significativas entre GC y GE.

CONCLUSIONES: Desde el diagnóstico se observan dificultades para la adaptación a la EP en pacientes y familiares, que podrían abordarse con intervenciones psicoeducativas multidisciplinares. 


\section{ABSTRACT}

TITLE: Psychoeducational intervention for people with Parkinson's disease and family carers: preliminary results at baseline time.

INTRODUCTION: The psychosocial adjustment influences on quality of life in patient with Parkinson's disease (PD) and his/her family carer. However, this is usually not addressed in clinical practice.

OBJECTIVE: To evaluate coping skills, psychosocial adjustment and quality of life in patients with PD and family carers from a quasiexperiment at baseline time.

METHODS: Quasi-experimental study carried out in Primary Care centres, with a sample of 80 patients with PD and 80 family carers, divided in a control group (CG) and an experimental group (EG). In the data collection was used the Psychosocial Adjustment Scale PAIS-SR, the Coping Scale Brief COPE and the Quality of Life Scales PDQ-39 and SQLC. The analysis of sociodemographic data and students $t$ tests is performed with SPSS 23.0.

RESULTS: In the CG the average age in patients was 73,2 years old, and in relatives 61,1 years old. In the EG the average age in patients was 74,8 years old, and in relatives 65,1 years old. In both groups, $54 \%$ and $40 \%$ of patients had Hoehn \& Yahr stage I. Patients and relatives from CG and EG noticed mild difficulties in the quality of life and psychosocial adjustment to illness. Patients and relatives from CG and EG used the evaluated coping skills with low frequency. No statistically significant differences were found between CG and EG.

CONCLUSION: Since the diagnosis some difficulties are observed for the adjustment to PD in patients and relatives which could be addressed with multidisciplinary psychoeducational interventions. 
PALABRAS CLAVES: intervención multidisciplinar; habilidades de afrontamiento; adaptación psicosocial; cuidadores informales; enfermedad de Parkinson.

KEY WORDS: multidisciplinary intervention; coping skills; psychosocial adjustment; informal caregivers; Parkinson's disease. 


\section{INTRODUCCIÓN}

La enfermedad de Parkinson (EP) es la segunda enfermedad neurodegenerativa más frecuente, con una prevalencia de $1 \%$ en la población mayor de 60 años de países desarrollados y 10 millones de personas afectadas a nivel mundial ${ }^{1,2}$. En España se estima que hay alrededor de 160.000 personas diagnosticadas de EP, y que el coste anual derivado de esta enfermedad asciende a 1.700 euros por persona ${ }^{3}$.

La EP puede provocar numerosos síntomas motores y no motores que afectan directamente en la calidad de vida de la persona ${ }^{4}$. A medida que la enfermedad progresa, la persona encuentra más dificultades para realizar sus actividades de la vida diaria, lo que favorece importantes cambios en su vida social ${ }^{5}$. En muchas ocasiones, estos cambios son percibidos por la persona como pérdidas en su vida impuestas por la EP que hacen muy complejo el afrontamiento de la enfermedad ${ }^{6}$. Sin embargo, varios estudios han evidenciado que la adaptación psicosocial a la enfermedad es un factor que influye de forma significativa en la calidad de vida de la persona con $\mathrm{EP}^{7,8}$, y que el afrontamiento puede predecir la adaptación psicosocial a la $E P^{8}$. Por ello se hace necesario que el sistema sanitario, además de abordar los síntomas de la EP, adopte estrategias para promover la adaptación psicosocial a la enfermedad en los pacientes con EP. Esas estrategias deben responder también a las necesidades de los familiares cuidadores de personas con EP dado que, en la mayoría de los casos, su implicación en el cuidado del paciente afecta a su bienestar y calidad de vida ${ }^{9,10}$. Actualmente en la EP, las intervenciones no farmacológicas que se han desarrollado para mejorar la calidad de vida no han abordado y evaluado la adaptación psicosocial y el afrontamiento de la EP, y tampoco han adoptado un enfoque de diada de pacientes con EP y familiares cuidadores ${ }^{6,9}$. Para dar respuesta a esta situación de la práctica clínica, en el Programa de investigación ReNACE se diseñó una intervención psicoeducativa multidisciplinar centrada en mejorar las habilidades de afrontamiento y la adaptación psicosocial en pacientes con EP y en sus familiares 
cuidadores. La efectividad de esta intervención se valorará a través de un cuasiexperimento con grupo control y grupo experimental.

El objetivo del presente estudio es evaluar las habilidades de afrontamiento, la adaptación psicosocial y la calidad de vida, en tiempo basal, en los participantes del grupo experimental y del grupo control del estudio.

\section{MÉTODOS}

Diseño

Estudio cuasiexperimental ${ }^{11}$ con grupo control y repetición de mediciones en Tiempo Basal (T0), Post-intervención (T1) y 6 meses post-intervención (T2). El grupo experimental recibirá una intervención psicoeducativa de 9 semanas, y el grupo control sesiones informativas durante 6 semanas. Con este diseño se pretende determinar el impacto a corto y a largo plazo en los participantes del grupo control e intervención en su calidad de vida, habilidades de afrontamiento y adaptación psicosocial a la enfermedad.

\section{Intervención experimental y grupo control}

La intervención ${ }^{11}$ que recibe el grupo experimental consiste en un programa psicoeducativo multidisciplinar de 9 semanas centrado en que los participantes desarrollen habilidades de afrontamiento que faciliten su adaptación psicosocial a la EP. Cada semana se organiza una sesión presencial de 90 minutos con 15-20 pacientes y otra sesión paralela, al mismo tiempo, con 15-20 cuidadores. La decisión de separar a los pacientes de sus familiares responde a la necesidad de abordar en cada sesión cuestiones específicas de cada grupo y también al interés de que los participantes compartan sus inquietudes sin miedo a preocupar a sus familiares. El enfoque de grupo se utiliza en todas las sesiones para intentar facilitar el intercambio 
de opiniones entre personas que viven una situación similar. Por ello, en cada sesión el profesional introduce un tema y al mismo tiempo lanza preguntas a los participantes, con la finalidad de que éstos reflexionen sobre su propia situación y las habilidades de afrontamiento que suelen utilizar. Los temas que se abordan en las 9 sesiones que constituyen la intervención y el profesional responsable de cada una se indica a continuación:

1. Introducción. Médico de Atención Primaria. Información sobre la dinámica de las sesiones y la importancia de participar e intercambiar opiniones.

2. Conocer la EP. Neurólogo. Temas: síntomas motores y no motores de la EP, tratamiento farmacológico y quirúrgico.

3. Hábitos de vida saludable. Enfermera. Temas: dieta, ejercicio físico, sueño/descanso y vida social.

4. El valor de la persona. Diversidad funcional y dependencia. Trabajadora social. Temas: reflexión sobre el valor que posee una persona; recursos disponibles y cómo solicitarlos.

5. La EP como compañera de viaje. Médico de Atención Primaria y Persona con EP. Temas: reflexión sobre la existencia del proceso de adaptación psicosocial a la enfermedad y de la importancia de las habilidades de afrontamiento para convivir mejor con la EP.

6. ¿Cuál es tu meta? Taller el espejo. Psicóloga. Temas: beneficios de la empatía, la paciencia y la autoestima positiva.

7. Conduce tu vida. Psicóloga. Temas: técnicas para relajarse en situación de estrés (respiración, gestión del tiempo, etc). 
8. ¿Qué quiere decir presente? Sonríe a la vida. Psicóloga. Temas: analizar la importancia de vivir el presente, participar en actividades de tiempo libre y las virtudes de la risa.

9. Conclusiones. Médico de Atención Primaria. Favorecer la integración de los temas analizados y reflexionar sobre los conocimientos, habilidades y actitudes trabajadas.

Los participantes del grupo control reciben sesiones informativas durante 5 semanas donde se tratan las características de la EP, la importancia de los hábitos de vida saludables, y se informa sobre los recursos que existen en la comunidad para las personas con alguna discapacidad y para sus cuidadores. Las sesiones también se realizan en grupos de 15-20 personas, separando pacientes y familiares en dos salas, y con una duración 90 minutos.

\begin{abstract}
Ámbito de estudio
En este estudio, que se inició en marzo del 2015, han participado seis centros de Atención Primaria del Servicio Navarro de Salud-Osasunbidea. De esta forma, este proyecto se realiza en el ámbito de la comunidad, puesto que es en este contexto donde esta población necesita contar con habilidades de afrontamiento que les ayuden en el proceso de convivencia con la EP. La selección del ámbito de la comunidad y de los centros de Atención Primaria coincide con las líneas de actuación definidas por la Estrategia para el Abordaje de la Cronicidad en el Sistema Nacional de Salud ${ }^{12}$, que subrayan Atención Primaria como referente para lograr una atención de calidad en la población con procesos crónicos.
\end{abstract}




\section{Participantes}

Los participantes de este estudio son personas diagnosticadas de EP, que reciben atención ambulatoria en los centros de salud seleccionados, mayores de 18 años, en cualquier estadio de la enfermedad, que residen en Navarra, y con capacidad cognitiva conservada determinada por su médico responsable.

También participan en este estudio los familiares/cuidadores de personas con EP, mayores de 18 años, residentes en Navarra, que conviven con el paciente o colaboren activamente en su cuidado.

El reclutamiento de pacientes y familiares es individual, es decir, no hay obligatoriedad de que participen la pareja de paciente y familiar, dada la importancia de incluir a pacientes sin familiar habitual y a familiares de pacientes que no pueden participar en el estudio por su deterioro cognitivo. Se excluirán aquellos participantes que no accedan voluntariamente a participar.

En este estudio se ha utilizado un muestreo de casos consecutivos en los centros participantes. La muestra actual es de 40 pacientes y 40 familiares en grupo control, y de 40 pacientes y 40 familiares en grupo experimental.

\section{Variables}

En este cuasiexperimento las variables son: Calidad de vida (variable dependiente); Habilidades de afrontamiento; Adaptación psicosocial a la enfermedad; Características sociodemográficas; y el estadio de la EP que se medirá sólo en los pacientes con la clasificación de Hoehn \& Yahr ${ }^{13}$.

\section{Recogida de datos}

La evaluación de los efectos de la intervención se mide con una recogida de datos en tres tiempos: Basal (pre-intervención), Post-intervención (justo después de terminar la intervención) y 6 meses post-intervención. Actualmente se ha realizado la recogida de 
datos del Tiempo Basal. Los instrumentos que se han utilizado con los participantes del GC y GE son los siguientes:

- Escala de Adaptación Psicosocial a la enfermedad (PAIS-SR) ${ }^{14}$. Se aplica en pacientes y cuidadores. Es una escala con 46 ítems. A mayor puntuación, mayores dificultades en la adaptación psicosocial a la enfermedad.

- Escala BRIEF COPE ${ }^{15,16}$. Está constituida por 24 ítems, y en cada ítem se indica del 1 a 4 con qué frecuencia suele utilizar cada habilidad de afrontamiento. Las puntuaciones altas indican mayor utilización de habilidades de afrontamiento. Se utiliza en los pacientes y en sus cuidadores.

- Escala Calidad de vida PDQ-39 ${ }^{17,18}$. Se utiliza para medir calidad de vida en los pacientes con EP. Está formada por 39 ítems. Puntuaciones altas indican mayor afectación en la calidad de vida.

- Escala Calidad de vida SQLC ${ }^{19,20}$. Esta escala tiene 16 ítems que miden el impacto que la enfermedad puede generar en la calidad de vida de los cuidadores. Las puntuaciones más bajas indican peor calidad de vida.

\section{Análisis de datos}

Se han realizado análisis descriptivos y de frecuencias de los datos sociodemográficos y de T-student para muestras independientes con el programa SPSS 23.0. El nivel de significación se estableció en 0.05 .

\section{Consideraciones éticas}

Este estudio ha sido aprobado por el Comité de Ética de la Universidad de Navarra y por los diferentes centros de Atención Primaria participantes. Todas las personas con EP y familiares cuidadores han participado de forma voluntaria en este estudio, tras ser informadas de las características del mismo y firmar un consentimiento informado escrito. Todos los datos de los participantes han sido tratados de forma confidencial. 


\section{RESULTADOS}

En este apartado se presentan los resultados de la medición a Tiempo Basal (antes de comenzar la intervención) en 40 pacientes y 40 familiares cuidadores del GC, y en 40 pacientes y 40 familiares cuidadores del GE. Los datos sociodemográficos de los participantes pueden consultarse en la Tabla 1.

En la medición basal se ha identificado que los pacientes (ver Tabla 2), por término medio, tienen una afectación leve de su calidad de vida, dado que presentan una puntuación total en la Escala PDQ-39 ${ }^{17,18}$ de 24,1 (D.E = 13,1) en el GC y de 21,6 (D.E $=14,0$ ) en el GE. Por su parte, en los familiares (ver Tabla 3) se ha observado también una afectación leve de su calidad de vida, al obtener por término medio una puntuación total en la Escala $S_{L L C}{ }^{19,20}$ de $104,3(D . E=25,3)$ en el GC y de 112,3 $(D . E=24,0)$ en el GE.

En relación a la variable de adaptación psicosocial, los resultados señalan que los pacientes y familiares del presente estudio perciben dificultades leves en su adaptación psicosocial a la enfermedad, porque en la Escala PAIS-SR ${ }^{14}$ presentan por término medio, los pacientes una puntuación total de $35,3(D . E=18,9)$ en el GC y 37,0 $(D \cdot E=20,0)$ en el GE; y los familiares de $25,8(D . E=13,4)$ en el $\mathrm{GC}$ y de 29,9 (D.E= 16,4) en el GE.

En la escala de afrontamiento BRIEF $\operatorname{COPE}^{15,16}$, los pacientes del GC y GE obtuvieron, por término medio, una puntuación total de $47,7(D . E=9,9)$ y $47,2(D . E=$ 9,5), en cambio, en los familiares se identificó una puntuación de 45,5 (D.E = 12,2) en el GC y de $45,1(D . E=10,5)$ en el GE. Teniendo en cuenta que la puntuación total máxima de la escala es 96 , cabe señalar que los participantes de este estudio no utilizan con mucha frecuencia las habilidades de afrontamiento evaluadas. De acuerdo con los resultados (ver Tabla 4), la habilidad de afrontamiento más utilizada por los pacientes del GC y del GE es la Aceptación con una puntuación de 6,2 $(D . E=1,7)$ en el GC y de 5,7 (D.E = 2,0) en el GE; y en segundo lugar el Uso del apoyo emocional, 
con una puntuación de 5,5 $(D . E=2,0)$ tanto en el GC como en el GE. En relación a los familiares, la habilidad de afrontamiento más utilizada es también la Aceptación, con una puntuación de $6,1(D . E=1,8)$ en el $G C$ y una puntuación de $6,3(D \cdot E=1,8)$ en el GE; $y$ en segundo lugar el Afrontamiento Activo con una puntuación de 5,2 $(D \cdot E=2,0)$ en el GC y una puntuación de 5,0 (D.E = 1,9) en el GE. Por el contrario, la habilidad de afrontamiento menos utilizada en ambos grupos fue el Uso de sustancias tóxicas, dado que los pacientes obtuvieron una puntuación de $2,1(D . E=0,2)$ en el GC y de 2,0 $(D \cdot E=0,0)$ en el GE; $y$ en los familiares, una puntuación de 2,0 $(D \cdot E=0,2)$ en el $\mathrm{GC}$ y de 2,3 $(D \cdot E=1,3)$ en el GE. Estos resultados indican que los pacientes y familiares utilizan con más frecuencia aquellas habilidades de afrontamiento que son positivas para su bienestar y adaptación a su situación.

En este estudio no se han identificado diferencias estadísticamente significativas entre el GC y GE en Calidad de vida, Adaptación psicosocial y Habilidades de afrontamiento (ver Tabla 2 y Tabla 3).

\section{DISCUSIÓN}

En este artículo se exponen las características basales de un grupo de pacientes con EP y familiares cuidadores que participaban en el GC y GE de un estudio centrado en evaluar una intervención psicoeducativa focalizada en potenciar el afrontamiento y la adaptación a la EP. Los resultados identificados señalan que los participantes del GC y del GE presentan características similares en la medición basal. Este hallazgo es positivo porque tener a una población con características comunes en ambos grupos, permitirá evaluar mejor el efecto que pueda tener la intervención psicoeducativa.

En este estudio, las personas con EP presentaban una afectación leve de su calidad de vida. Este hallazgo podría ser debido a que alrededor del $50 \%$ de los participantes se encontraba en el estadio I de la EP según la clasificación de Hoehn \& Yahr ${ }^{13}$. Este resultado coincide con investigaciones anteriores que evidencian que los pacientes 
con EP tienen peor calidad de vida conforme aumenta la severidad de su enfermedad y los síntomas motores y no motores ${ }^{4}$. De ahí que los participantes de este estudio, que se encontraban en un estadio inicial, tuviesen una afectación leve de su calidad de vida. Por tanto, es esencial que el sistema sanitario garantice desde el diagnóstico de la EP, una adecuada valoración y atención de los síntomas de la EP, y especialmente de los síntomas no motores, como el cansancio, los trastornos del sueño o los cambios en el estado de ánimo, dado que estos pueden producir un notable impacto en la calidad de vida de las personas con EP, incluso mayor al que ejercen los síntomas motores ${ }^{4}$.

Asimismo, en este estudio se identificó que los familiares cuidadores también tenían una afectación leve de su calidad de vida. Es importante subrayar este resultado, dado que en muchas ocasiones, la asistencia sanitaria no ofrece una adecuada atención a los familiares cuidadores de personas con EP. Sin embargo, la implicación de los familiares en el cuidado de la persona con EP conlleva con frecuencia cambios en los roles del familiar que pueden afectar a su bienestar y calidad de vida ${ }^{9,10}$. Por tanto, es necesario que el sistema sanitario reconozca y facilite intervenciones que desarrollen promoción de la salud y prevención de enfermedades en los familiares cuidadores de personas con EP, similares a la intervención descrita en este artículo, que respondan a las necesidades no cubiertas de los familiares y ayuden a disminuir su estrés ${ }^{9,10}$.

Los hallazgos del presente estudio señalan que las personas con EP y los familiares cuidadores percibían dificultades leves en su adaptación psicosocial a la enfermedad. Esto podría estar relacionado con el hecho de que la mayoría de los pacientes con EP se encontraban en estadios iniciales de la enfermedad, y a que generalmente los síntomas de la EP se presentan de forma gradual, lo que puede facilitar la adaptación a los mismos ${ }^{21}$. Por otra parte, el haber identificado dificultades leves en la adaptación psicosocial a la EP podría estar relacionado con que los participantes utilizaban de forma más frecuente habilidades de afrontamiento que generalmente han sido 
asociadas con una adaptación positiva, como es el Uso de apoyo social, la Aceptación o el Afrontamiento Activo ${ }^{22}$. Sin embargo, en general se ha identificado que los participantes utilizaban las habilidades de afrontamiento con baja frecuencia, puesto que en la mayoría de éstas obtuvieron una puntuación de alrededor de 4, cuando la puntuación máxima es 8. Por ello, es importante que desde atención primaria se desarrollen intervenciones que aborden la afectación psicológica y social que supone convivir con EP desde el del diagnóstico. Estas intervenciones pueden facilitar a los pacientes con EP y a sus familiares cuidadores información que les ayude a comprender mejor sus cambios psicosociales, y además, pueden servir como espacios para la reflexión sobre la importancia de su actitud en el cuidado de su salud y de la EP. De esta manera, cuando la EP progresa y aparecen más síntomas, el paciente y el familiar cuidador podrían sentirse más preparados para adaptarse a la nueva situación y continuar experimentando un sentimiento de normalidad en sus vidas ${ }^{23}$.

En relación a las limitaciones de este estudio, señalar que el carácter voluntario de los participantes impide la generalización de sus resultados. Además, es preciso obtener un mayor tamaño muestral para poder evaluar posteriormente el efecto de la intervención psicoeducativa. Por último, esta investigación debería llevarse a cabo en otras poblaciones, dado que los resultados pueden estar influidos por el contexto de los participantes.

En conclusión, destacar que desde el inicio de la EP, tanto en pacientes como en sus familiares cuidadores, se observan algunas dificultades en la adaptación psicosocial a la enfermedad y un impacto leve en su calidad de vida. Por ello, es esencial que en la práctica clínica se adopten intervenciones que contemplen el impacto emocional y los cambios sociales que derivan de la EP, para que unido al tratamiento de los síntomas físicos, mejoren la atención de los pacientes y las familias que conviven con EP. 


\section{CONFLICTO DE INTERESES}

Los autores declaran no tener ningún conflicto de intereses.

\section{BIBLIOGRAFÍA}

1. Mateus C, Coloma J. Health economics and cost of illness in Parkinson's disease. Eur Neurol Rev. 2013; 8: 6-9.

2. European Parkinson's Disease Association (EPDA). About Parkinson's. (Acceso 9 abril 2018). Disponible en: http://www.epda.eu.com/about-parkinson-s/what-isparkinson-s/ (2018).

3. Peñas Domingo E, Gálvez Sierra M, Marín Valero M, Pérez-Olivares Castiñeira M. Libro Blanco del Parkinson en España. Aproximación, análisis y propuesta de futuro. 2015; Madrid: Ministerio de Sanidad, Servicios Sociales e Igualdad. Federación Española de Parkinson.

4. Prakash KM, Nadkarni NV, Lye W-K, Yong M-H, Tan E-K. The impact of non-motor symptoms on the quality of life of Parkinson's disease patients: a longitudinal study. Eur J Neurol. 2016; 23(5): 854-860. doi:10.1111/ene.12950

5. Wressle E, Engstrand C, Granérus AK. Living with Parkinson's disease: elderly patients' and relatives' perspectives on daily living. Australian Occupational Therapy Journal. 2007; 54: 131-139. doi: 10.1111/j.1440-1630.2006.00610.x

6. Navarta-Sánchez MV, Caparrós N, Riverol M, Díaz de Cerio S, Ursúa ME, Portillo MC. Core elements to understand and improve coping with Parkinson's disease in patients and family carers: a focus group study. J Adv Nurs. 2017; 73: 2609-2621. doi: 10.1111/jan.13335 
7. Suzukamo Y, Ohbu S, Kondo T, Kohmoto J, Fukuhara S. Psychosocial adjustment has a greater effect on health-related quality of life than on severity of disease in Parkinson's disease. Mov Disord. 2006; 21:761-766. doi: 10.1002/mds.20817.

8. Navarta-Sánchez MV, Senosiain García JM, Riverol M, Ursúa Sesma ME, Díaz de Cerio Ayesa S, Anaut Bravo S, et al. Factors influencing psychosocial adjustment and quality of life in Parkinson patients and informal caregivers. Qual Life Res. 2016; 25:1959-1968. doi: 10.1007/s11136-015-1220-3

9. Mosley PE, Moodie R, Dissanayaka N. Caregiver Burden in Parkinson Disease: A Critical Review of Recent Literature. J Geriatr Psychiatry Neurol. 2017; 30(5): 235-252. doi: $10.1177 / 0891988717720302$

10. Lageman SK, Mickens MN, Cash TV. Caregiver-identified needs and barriers to care in Parkinson's disease. Geriatr Nurs. 2015; 36 (3): 197-201. https://doi.org/10.1016/j.gerinurse.2015.01.002

11. Navarta-Sánchez MV, Ursua ME, Riverol M, Ambrosio L, Álvarez MJ, Díaz de Cerio S, et al. (2018). Implementation of a multidisciplinary psychoeducational intervention for Parkinson's disease patients and carers in the community: study protocol. BMC Fam pract. 19:45. https://doi.org/10.1186/s12875-018-0730-9

12. Ministerio de Sanidad, Servicios Sociales e Igualdad. Estrategia para el Abordaje de la Cronicidad en el Sistema Nacional de Salud. 2012. (Acceso 9 abril 2018). Disponible

en: http://www.msssi.gob.es/organizacion/sns/planCalidadSNS/pdf/ESTRATEGIA ABORD AJE CRONICIDAD.pdf

13. Hoehn MM, Yahr MD. Parkinsonism: onset, progression and mortality. Neurology. $1967 ; 17(5): 427-42$. 
14. Derogatis LR. The Psychosocial adjustment to illness scale (PAIS). J Psychosom Res. 1986;30:77-91.

15. Carver CS. You want to measure coping but your protocol's too long: consider the Brief COPE. Int J Behav Med 1997;4:92-100. doi: 10.1207/s15327558ijbm0401_6.

16. Perczek R, Carver CS, Price AA. Coping, mood and aspects of personality in Spanish translation and evidence of convergence with English versions. J Pers Assess 2000; 74: 63-87. doi: 10.1207/S15327752JPA740105

17. Peto V, Jenkinson C, Fitzpatrick R, Greenhall R. The Development and Validation of a Short Measure of Functioning and Well Being for Individuals with Parkinson's Disease. Qual Life Res. 1995;4:241-48.

18. Martínez-Martín P, Frades Payo B \& The Grupo Centro for Study of Movement Disorders. Quality of life in Parkinson's disease: validation study of the PDQ-39 Spanish version. J Neurol. 1998;245(1):34-38.

19. Glozman JM, Bicheva KG, Fedorova NV. Scale of Quality of Life of Care-Givers (SQLC). J Neurol 1998;245(Suppl 1):S39-S41. doi: 10.1007/PL00007738

20. Martínez-Martín P, Guerrero MT, Frades B, Fontán C, Glozman JM. Quality of life of caregivers: a new perspective on the consequences of Parkison's disease. Mov Disord 1998;13(Suppl 2):p63. ISSN: 1531-8257.

21. Zaragoza Salcedo A, Senosiain García JM, Riverol Fernández M, Anaut Bravo S, Díaz de Cerio Ayesa S, Ursúa Sesma ME, et al. Elementos claves en el proceso de convivencia con la enfermedad de Parkinson de pacientes y familiares cuidadores. [Key elements in the process of living with Parkinson's disease for patients and caregivers]. An Sist Sanit Navar 2014;37:69-80. doi: 10.4321/S113766272014000100008 . 
22. Stanton AL, Revenson TA, Tenneh H. Health Psychological Adjustment to Chronic Disease. Annu Rev Psychol. 2007;58:565-592.

23. Boehmer KR, Gionfriddo MR, Rodriguez-Gutierrez R, Abu Dabrh AM, Leppin AL, Hargraves I, et al. (2016). Patient capacity and constraints in the experience of chronic disease: a qualitative systematic review and thematic synthesis. BMC Fam Pract. 2016; 17(127): 1-23. doi: 10.1186/s12875-016-0525-9 
TABLAS

Tabla 1. Características sociodemográficas de los participantes.

\begin{tabular}{lcccc}
\hline & \multicolumn{2}{c}{ GRUPO CONTROL } & \multicolumn{2}{c}{ GRUPO EXPERIMENTAL } \\
& PACIENTES & CUIDADORES & PACIENTES & CUIDADORES \\
\hline Edad & 73,2 & 61,1 & 74,8 & 65,1 \\
Género & & & & \\
Hombre & $65 \%$ & $15 \%$ & $60 \%$ & $13 \%$ \\
Mujer & $35 \%$ & $85 \%$ & $40 \%$ & $87 \%$ \\
Estudios básicos & $72,5 \%$ & $40 \%$ & $70 \%$ & $65 \%$ \\
Formación profesional & $2,5 \%$ & $17,5 \%$ & $7,5 \%$ & $10 \%$ \\
Estudios universitarios & $25 \%$ & $42,5 \%$ & $22,5 \%$ & $25 \%$ \\
Estadio I Hoehn \& Yahr & $54 \%$ & - & $40 \%$ & - \\
\hline
\end{tabular}


Tabla 2. Resultados de la medición a tiempo basal en los pacientes.

\begin{tabular}{|c|c|c|c|c|c|}
\hline RESULTAD & SS EN PACIEN & ON & RMEDA & E PAF & INSON \\
\hline & Grupo & $\mathbf{N}$ & Media & D.E. & T student $(p)$ \\
\hline \multirow{3}{*}{ Edad } & Control & 40 & 73,2 & 7,3 & \multirow{3}{*}{$-0,824(0,413)$} \\
\hline & & & & & \\
\hline & Experimental & 40 & 74,8 & 9,0 & \\
\hline \multirow{2}{*}{$\begin{array}{l}\text { CALIDAD DE VIDA } \\
\text { PDQ39 }\end{array}$} & Control & 40 & 24,1 & 13,1 & \multirow[t]{2}{*}{$0,827(0,411)$} \\
\hline & Experimental & 40 & 21,6 & 14,0 & \\
\hline \multirow{2}{*}{$\begin{array}{l}\text { ADAPTACIÓN } \\
\text { PAIS-SR }\end{array}$} & Control & 40 & 35,3 & 18,9 & \multirow[t]{2}{*}{$-0,401(0,689)$} \\
\hline & Experimental & 40 & 37,0 & 20,0 & \\
\hline \multirow{2}{*}{$\begin{array}{l}\text { AFRONTAMIENTO } \\
\text { BRIEF COPE }\end{array}$} & Control & 40 & 47,7 & 9,9 & \multirow{2}{*}{$0,252(0,802)$} \\
\hline & Experimental & 40 & 47,2 & 9,5 & \\
\hline
\end{tabular}

D. E.= Desviación Estándar 
Tabla 3. Resultados de la medición a tiempo basal en los familiares cuidadores.

\begin{tabular}{|c|c|c|c|c|c|}
\hline \multicolumn{6}{|c|}{ RESULTADOS EN FAMILIARES CUIDADORES } \\
\hline & Grupo & $\mathbf{N}$ & Media & D.E. & T student $(p)$ \\
\hline \multirow{3}{*}{ Edad } & Control & 40 & 61,1 & 14,6 & \multirow{3}{*}{$-1,327(0,189)$} \\
\hline & & & & & \\
\hline & Experimental & 40 & 65,1 & 12,4 & \\
\hline \multirow{2}{*}{$\begin{array}{l}\text { CALIDAD DE VIDA } \\
\text { SQLC }\end{array}$} & Control & 40 & 104,3 & 25,3 & \multirow{2}{*}{$-1,449(0,151)$} \\
\hline & Experimental & 40 & 112,3 & 24,0 & \\
\hline \multirow{2}{*}{$\begin{array}{l}\text { ADAPTACIÓN } \\
\text { PAIS }\end{array}$} & Control & 40 & 25,8 & 13,4 & \multirow[b]{2}{*}{$-1,207(0,231)$} \\
\hline & Experimental & 40 & 29,9 & 16,4 & \\
\hline \multirow{3}{*}{$\begin{array}{l}\text { AFRONTAMIENTO } \\
\text { BRIEF COPE }\end{array}$} & Control & 40 & 45,5 & 12,2 & \multirow{3}{*}{$0,157(0,876)$} \\
\hline & & & & & \\
\hline & Experimental & 40 & 45,1 & 10,5 & \\
\hline
\end{tabular}

D. E.= Desviación Estándar 
Tabla 4. Resultados en la Escala de Afrontamiento BRIEF COPE en tiempo basal.

\begin{tabular}{|c|c|c|c|}
\hline & Grupo & PACIENTES $^{1}$ & CUIDADORES $^{1}$ \\
\hline \multirow{2}{*}{ Auto distracción } & Control & $4,2(1,7)$ & $3,4(1,7)$ \\
\hline & Experimental & $3,7(1,7)$ & $3,6(1,7)$ \\
\hline \multirow{2}{*}{$\begin{array}{l}\text { Afrontamiento } \\
\text { activo }\end{array}$} & Control & $4,4(1,7)$ & $5,2(2,0)$ \\
\hline & Experimental & $4,8(1,8)$ & $5,0(1,9)$ \\
\hline \multirow{2}{*}{ Negación } & Control & $2,9(1,2)$ & $2,5(1,1)$ \\
\hline & Experimental & $3,0(1,3)$ & $2,4(0,9)$ \\
\hline \multirow{2}{*}{$\begin{array}{l}\text { Uso de sustancias } \\
\text { tóxicas }\end{array}$} & Control & $2,1(0,2)$ & $2,0(0,2)$ \\
\hline & Experimental & $2,0(0,0)$ & $2,3(1,3)$ \\
\hline \multirow{2}{*}{$\begin{array}{l}\text { Uso de apoyo } \\
\text { emocional }\end{array}$} & Control & $5,5(2,0)$ & $4,0(2,1)$ \\
\hline & Experimental & $5,5(2,0)$ & $4,3(2,2)$ \\
\hline \multirow{2}{*}{$\begin{array}{l}\text { Desvinculación de } \\
\text { comportamiento }\end{array}$} & Control & $2,8(1,4)$ & $2,5(1,1)$ \\
\hline & Experimental & $2,8(1,2)$ & $2,6(1,5)$ \\
\hline \multirow{2}{*}{ Descarga } & Control & $3,6(1,8)$ & $3,6(1,4)$ \\
\hline & Experimental & $3,5(1,7)$ & $3,2(1,5)$ \\
\hline \multirow{2}{*}{ Positividad } & Control & $4,4(1,9)$ & $4,6(1,7)$ \\
\hline & Experimental & $4,4(1,7)$ & $4,4(1,8)$ \\
\hline \multirow{2}{*}{ Planificación } & Control & $4,0(1,8)$ & $5,0(1,8)$ \\
\hline & Experimental & $4,6(1,7)$ & $4,4(2,0)$ \\
\hline \multirow{2}{*}{ Humor } & Control & $3,3(2,0)$ & $3,1(1,7)$ \\
\hline & Experimental & $2,9(1,5)$ & $2,4(1,1)$ \\
\hline \multirow{2}{*}{ Aceptación } & Control & $6,2(1,7)$ & $6,1(1,8)$ \\
\hline & Experimental & $5,7(2,0)$ & $6,3(1,8)$ \\
\hline \multirow[b]{2}{*}{ Religión } & Control & $4,5(2,4)$ & $4,8(2,4)$ \\
\hline & Experimental & $4,3(2,1)$ & $4,8(2,3)$ \\
\hline
\end{tabular}


1

2

3

4

5

9

10

11

12

13

14

15

16

17

18

19

20

21

22

23

24

25

26

27

28

29

30

31

32

33

34

35

36

37

38

39

40

41

42

43

44

45

46

47

48

49

50

51

52

53

54

55

56

57

58

59

60

61

62

63

64

65 